\title{
DO SKEUOMORPHISM AO FLAT DESIGN: A FORMAÇÃO DE TENDÊNCIAS ESTÉTICAS SOB A ÓTICA DO DESIGN DE INTERFACES
}

\author{
Jéssica Rodrigues Esteves \\ Universidade Federal de Pelotas \\ jess.re@gmail.com \\ Tobias Mülling \\ Universidade Federal de Pelotas \\ tmulling@gmail.com
}

Resumo: O presente artigo apresenta o resultado de uma pesquisa sobre a formação de tendências estéticas no design de interfaces, compreendendo o Skeuomorphism e o Flat Design. Ambas são tendências utilizadas no design de interface como forma de referencial estético para a criação de um projeto e se diferem quanto uso de metáforas visuais, identificadas pela quantidade de elementos utilizados para a criação da interface. A fim de contextualizar as tendências estéticas abordadas, foi realizada uma revisão bibliográfica sobre os temas relevantes à pesquisa - conceituação de tendência, Skeumorphism e Flat Design; uma breve abordagem sobre a história das primeiras interfaces gráficas; e uma análise da interface do aplicativo "Notas" presente no sistema operacional iOS da Apple. A análise da interface do aplicativo "Notas" em diferentes versões de atualização do iOS permite a identificação de uma transição de tendências estéticas: no iOS 6 a estética do aplicativo assemelhava-se ao Skeuomorphism, enquanto nas versões atuais - a partir do iOS 7 - a interface representa o Flat Design. A análise compreendeu a observação de elementos como: tipografia, cor, legibilidade, iconografia e interação. Desta forma, foi possível caracterizar ambas tendências, destacando as principais diferenças entre o Skeumorphism e o Flat Design.

Palavras-chave: Design de Interfaces, Tendências, Skeuomorphism, Flat Design.

Abstract: This paper presents the results of a research about the formation of aesthetics trends in interface design, including Skeuomorphism and Flat Design. Skeumorphism and Flat Design are trends used on interfaces as the aesthetics referential to design a project. Both trends provides different visual metaphors and elements to design an interface. In order to contextualize the visual trends, a review of the relevant research themes was conducted, including a study about trend concepts, Skeuomorphism, and Flat design; a brief approach to the graphic user interface history and an analysis about "Notes", a native iOS's application. Analyzing the 
application "Notes" in different iOS versions an aesthetic transition occurred: in the iOS 6 the application aesthetics resembled Skeuomorphism while in the current versions - starting from iOS 7 - the interface features the Flat Design trend. The analysis involved the observation of design elements such as typography, color, legibility, iconography, and interaction. Thus, it was possible to characterize both trends, highlighting the main differences between the Skeuomorphism and the Flat Design.

Keywords: Interface design, trends, Skeuomorphism, Flat Design.

\section{INTRODUÇÃO}

As tendências estão presentes no design e influenciam a criação de novos produtos e serviços. Além de influenciar a questão técnica e mercadológica de um projeto de design, as tendências influenciam também a aparência estética. No design de interfaces, observam-se predominantemente a utilização de duas tendências estéticas: o Skeuomorphism e o Flat Design. O Skeuomorphism é uma estética visual que utiliza ornamentos para representar objetos da vida real. Esta estética faz uso de metáforas e affordances (NORMAN, 2006) com o objetivo de facilitar o entendimento do usuário sobre uma determinada tarefa ou funcionalidade.

No Skeuomorphism, os objetos buscam relação com o ambiente analógico, não somente pela metáfora em relação a funcionalidade, mas especialmente nos detalhes estéticos e assimilação com o ambiente real. Este tipo de relação é identificada como uma tendência no design de interfaces, utilizada desde o desenvolvimento das primeiras interfaces gráficas. Com a evolução das interfaces gráficas grande parte dos usuários foi adquirindo um vasto repertório de elementos do meio digital. Desta forma, os usuários não precisam mais visualizar as obviedades dos controles, ou seja, não necessitam que um website de um jornal possua a mesma aparência estética de um jornal físico para compreender a informação. Além disso, no nosso dia a dia lidamos com um fluxo de informação muito grande tornando-se exaustivo avaliar, filtrar e reter a informação; sobretudo nos dispositivos móveis. Portanto, uma interface com menos elementos irá auxiliar a filtrar a informação, focando no que é realmente importante e reduzindo também a desordem visual. Este cenário faz com que seja necessária a utilização de uma nova tendência estética, que priorize a transmissão da informação de maneira clara e rápida. Assim, surge o Flat Design, uma tendência visual contemporânea caracterizada pela simplificação dos elementos de interface com foco na forma, funcionalidade e tecnologia.

A fim de compreender as duas tendências e suas características foi realizada uma análise das interfaces dos sistemas operacionais móveis iOS 6 e iOS 7 da Apple. A partir da observação entre os dois sistemas analisou-se as mudanças relativas a estética, usabilidade e interação do aplicativo "Notas". Com base na pesquisa e análise tornou-se possível perceber empiricamente a eficácia das diferentes tendências e suas características visuais, sob a premissa da informação estética. 


\section{DESENVOLVIMENTO}

A metodologia utilizada para a elaboração desta pesquisa consistiu no método indutivo com abordagem qualitativa, realizada a partir de revisão bibliográfica e análises. Segundo Lakatos e Marconi (2010), o método indutivo obtém conclusões gerais a partir de premissas individuais. Esse método parte de dados particulares para inferir uma verdade geral ou universal, não contida nas partes examinadas. Desta forma, o objetivo dos argumentos indutivos é "levar a conclusões cujo conteúdo é muito mais amplo do que o das premissas nas quais se basearam" (Ibid., p. 85).

A revisão bibliográfica compreendeu assuntos como a conceituação de tendências, abordando a definição de autores como Caldas (2004), Raymond (2010), Picoli (2009, p. 18 apud Morace, 2007) e Vejlgaard (2007); a utilização do Modelo de Tendências em Forma de Diamante de Vejlgaard (2007); uma abordagem sobre o Skeuomorphism, tendo como base o conceito de affordances de Norman (2006); e um breve estudo sobre o Flat Design, reunindo acontecimentos que podem ter contribuído para sua popularização. Para a contextualização das tendências estéticas abordadas foi realizada uma análise do aplicativo "Notas" em diferentes versões de atualização do sistema operacional iOS da Apple, sendo eles iOS 6 e iOS 7. Embora o sistema operacional mais recente seja o iOS 9, a transição de uma interface que apresentava a tendência estética do skeuomorphism para uma interface flat ocorreu entre as atualizações do iOS 6 para o iOS 7. Com o objetivo de identificar as tendências estéticas, foram analisados elementos como tipografia, cor, legibilidade, iconografia, forma e interação. Esta análise tem o objetivo de mostrar as principais mudanças entre as duas tendências estéticas do design de interface.

\subsection{Conceituando tendência}

Vivemos em uma sociedade consumista, em que a oferta excede geralmente a procura, os produtos são normalizados e os padrões de consumo estão massificados. Ao consumir determinados produtos grande parte das pessoas têm preferência por aqueles que estejam na moda: desde produtos mais comuns, como acessórios e bijuterias; até os de alto investimento, como smartphones e carros. Estar na moda significa consumir o que há de novo e inovador no mercado. As tendências podem ser compreendidas como novidades existentes no mercado, ou seja, acontecimentos que mudaram o paradigma atual. Ao consumirmos um produto inovador e irreverente no mercado estamos sendo influenciados por tendências.

Quais são as tendências do mercado eletrônico, da próxima coleção de moda, do design contemporâneo? Na cultura contemporânea, a noção de tendência se faz presente em nosso cotidiano. A palavra tendência vem do termo em latim tendentia, particípio presente e nome plural substantivado do verbo tendere, que significa "tender para", "inclinar-se para" ou "atraído por" (CALDAS, 2004). Raymond (2010) comenta que o termo também era utilizado na antiga Alemanha e poderia significar "rodar" ou "girar". Já Caldas (2004, p. 22), acredita que na sociedade contemporânea esse conceito "foi construído com base nas ideias de movimento, mudança, representação do futuro, evolução e sobre critérios quantitativos". Picoli (2009, p. 18 apud Morace, 2007) considera o conceito de tendência complexo, no entanto, é possível considerar que uma tendência indica o surgimento de novos elementos no sistema sociocultural, caracterizando-se como um fenômeno de evolução. 
No final do século $X X$, o uso da palavra tendência tornou-se comum pela indústria de moda, e foi assim que a expressão começou a fazer parte do dia a dia de grande parte das pessoas. De acordo com o Vejlgaard (2007), revistas especializadas podem usar tendência como definição para novos produtos. Pessoas relacionadas ao design podem usar o termo para falar do desenvolvimento de algum objeto. Já um sociólogo que estuda este conceito afirmará que tendência é algo que não ocorreu, mas sim uma previsão de alguma coisa que irá acontecer de maneira específica e que afetará a maioria das pessoas; ou seja, um processo de mudança causado perante a sociedade.

Na moda as tendências são o resultado de estudos, pesquisas e análises de fenômenos e acontecimentos sociais que vêm das ruas, como também de diversas áreas como economia, política, sociologia, ciência e tecnologia. São sinais que anunciam o que vai acontecer num determinado espaço de tempo e isso interfere no consumo e no comportamento das pessoas, direcionando desejos e gostos (Vejlgaard, 2007).

As tendências para determinada estação são lançadas através de desfiles em eventos de moda, como o SPFW (São Paulo Fashion Week). A moda das passarelas não será exatamente a que será comercializada para a população: na maior parte das vezes, ela será adaptada para uma realidade urbana; a moda é apenas um conceito, que envolve cores, padrões, texturas e tecidos que serão a tendência da estação. A moda das passarelas poderá influenciar outros setores criativos, como o design de produto, o cinema e até mesmo o design de interfaces. Já no design de interfaces, pode-se identificar tendências a partir de mudanças em padrões de design por produtos que possuam relevância e notoriedade no mercado.

As tendências do design de interface difundem-se em sites de referências, como Blogs, Tumblr, Pinterest, Behance e Cargo Collective. A formação de tendências não obedece a uma metodologia específica. Entretanto, a utilização de metodologias faz com que seja possível identificar como ocorre a sua difusão. Nesta pesquisa, será utilizado o Modelo de Tendências em Forma de Diamante (VEJLGAARD, 2007) devido a sua categorização de grupos sociais que formam tendências, tornando-se possível adaptá-lo sob a ótica do design de interfaces.

O Modelo de Tendências em Forma de Diamante foi criado por Henrik Vejlgaard (2007), pioneiro no estudo de sociologia da tendência. Este modelo consiste em definir principalmente oito perfis diferentes de personalidade, como pode-se na observar na figura 1: catalizadores de tendências (trend creators), precursores de tendências (trendsetters), seguidores de tendências (trend followers), primeira maioria (early mainstreamers), grande maioria (mainstreamers), maioria tardia (late mainstreamers), conservadores (conservatives) e os anti-inovadores (anti innovation). 


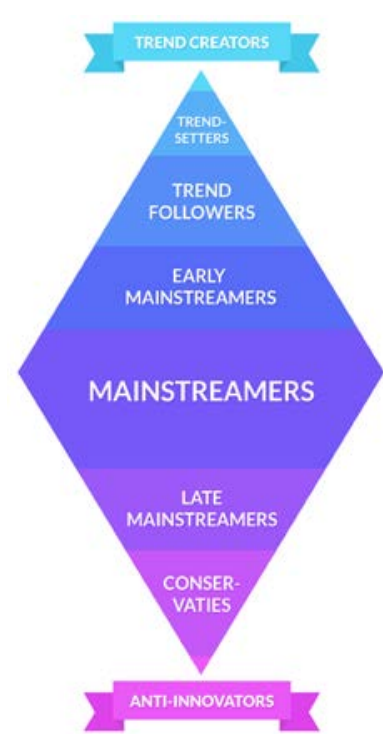

Figura 1 - Modelo de Tendências em Forma de Diamante.

Fonte: Adaptado de VEJLGAARD (2007, p.9).

Este modelo divide as pessoas de acordo com os grupos de tendências, definidos por seu processo de aceitação da tendência. No topo do modelo, encontramse os trend creators seguidos pelos trendsetters. Os primeiros são um grupo extremamente pequeno e heterogêneo de pessoas. É praticamente impossível definir quem são trend creators, no entanto, entende-se que as tendências partem deste grupo e atingem os trendsetters. Estes iniciam as tendências, ou seja, são formadores de opinião. Embora não sejam os criadores da tendência, conseguem exercer uma grande influência na sociedade e difundí-la aos demais grupos. Dando sequência a divisão do modelo, existem os early mainstreamers e os mainstreamers. Os early mainstreamers aceitam os novos estilos antes da maioria, antes que os estilos se tornem completamente mainstream; já os mainstreamers são a maioria que aceitam as tendências. Os mainstreamers podem ser definidos pelo lema: "não seja o primeiro a tentar o novo e não seja o último deixar o velho" (VEJLGAARD, 2007, tradução da autora). Os demais grupos - late mainstreamers, conservativeis e anti-innovators são os mais hesitantes em aceitar novidades. Estes grupos só irão aceitar as tendências quando não é mais possível ir contra ela.

O foco deste trabalho é analisar as tendências estéticas utilizadas no design de interfaces. Desta forma, pode-se identificar que o Flat Design aparenta estar representado atualmente pelos mainstreamers, tendo em vista que essa tendência está sendo utilizada em grande escala por designers.

\subsection{Skeuomorphism}

O Skeuomorphism é um termo que define um princípio de design que utiliza ornamentos ou detalhes para representar objetos físicos da vida real. A palavra é

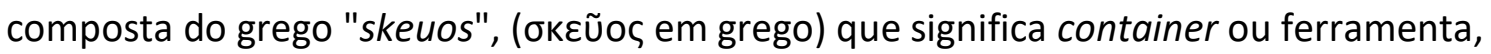
e morphê, ( $\mu$ opфń em grego) que é forma. Do ponto de vista estético do design de interfaces, o Skeuomorphism é representado pelo uso de sombras, gradientes, 
texturas, efeitos 3D e o que mais for necessário para familiarizar o usuário com a interface através de elementos do mundo real.

O Skeuomorphism também pode ser compreendido utilizando os conceitos de metáfora e affordances (NORMAN, 2006). Embora sejam dois conceitos distintos, ambos referem-se a utilização de propriedades reais do objeto para mostrar indicações de como usá-lo. Essas características são observadas no design de interfaces, que faz uso de ícones, janelas, menus, ponteiros, cores e outros elementos gráficos para familiarizar o usuário com o sistema.

Para Norman, o termo affordance refere-se "às propriedades percebidas e reais de um objeto, principalmente as propriedades fundamentais que determinam de que maneira o objeto poderia ser usado" (NORMAN, 2006, p. 33). Diariamente utilizamos a affordance dos objetos de forma inconsciente: giramos maçanetas e torneiras, abrimos e fechamos portas, utilizamos facas para cortar, apertamos um interruptor para acender uma lâmpada. Quando se tira proveito das affordances, o usuário sabe o que fazer apenas ao olhar: não são necessárias imagens ilustrativas, rótulos ou instruções. Objetos complexos podem exigir explicações, mas objetos simples não devem precisar delas. Quando objetos simples precisam de imagens, rótulos ou instruções, o design fracassou (NORMAN, 2006)

No design de interfaces as affordances podem ser representadas por elementos de interação como ícones, botões, links, janelas e menus. Esses elementos "falam por si", ou seja, sugerem uma ideia de ação. Por exemplo: quando acessamos um site de compras, normalmente nos deparamos com um botão destacado com o texto "comprar" ou com um ícone de um carrinho de compras. Sabemos que ao pressionar o botão iremos realizar a ação de comprar o item, devido ao modelo conceitual que possuímos desses elementos. Mas isso nem sempre foi assim: a evolução das interfaces gráficas para o usuário $\left(\mathrm{GUI}^{1}\right)$ nos ensinou e reforçou os modelos conceituais, que serão explicados a seguir.

Ao analisarmos a história das primeiras interfaces gráficas, nos deparamos com a utilização de metáforas e affordances para familiarizar os usuários com os computadores. O primeiro computador que utilizou a metáfora de desktop e uma GUI ${ }^{1}$ foi o Alto Personal Computer, desenvolvido pela Xerox PARC em 1973 (ROYO, 2008). Este computador não foi desenvolvido para ser um produto comercial, mas milhares de unidades foram construídas e utilizadas em escritórios da Xerox. A interface gráfica possuía janelas, ícones e menus para utilizar comandos como abrir, apagar e mover arquivos. Em 1975, foram implementadas inovações, como o uso de ícones e de menus pop-up ${ }^{2}$. Em 1981, a Xerox lançou o Star (fig.2), incorporando muitas das inovações do Alto Personal Computer. Apesar de não ser bem sucedido comercialmente, o Star serviu como referência para o desenvolvimento de sistemas posteriores, como os projetados pelas empresas Apple, Microsoft e Sun Microsystems.

\footnotetext{
${ }^{1}$ Interface gráfica para o usuário (do inglês, Graphical User Interface). Pode ser compreendido como um tipo de interface do utilizador que permite a interação com dispositivos digitais através de elementos gráficos como ícones e outros indicadores visuais.

2 o pop-up é uma janela extra que abre no navegador ao visitar um website ou acessar uma hiperligação específica.
} 


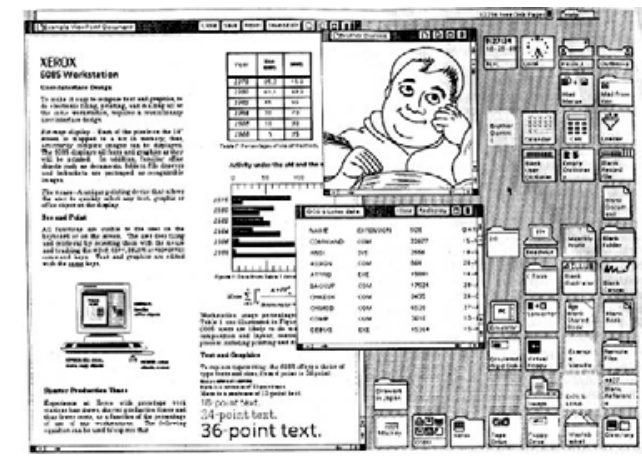

Figura 2 - Interface Gráfica da Xerox Star.

Fonte: (http://zip.net/bkncp1)

Em 1984, a Apple lançou o Macintosh, o primeiro produto bem sucedido comercialmente que utilizou uma GUI de vários painéis, como podemos observar na figura 3. A metáfora de desktop foi utilizada: os arquivos pareciam pedaços de papel, diretórios de arquivos pareciam pastas de arquivo. O desktop ainda possuía um conjunto de acessórios para o usuário distribuir ao redor da tela como calculadora, bloco de notas e despertador. Caso desejasse apagar arquivos ou pastas, o usuário poderia arrastá-los para a lixeira. Royo (2008) comenta que o lançamento deste computador fez com que a metáfora de desktop se tornasse popular. De acordo com o autor, "o fato em si foi muito importante porque envolveu a mudança na mentalidade de muitos usuários" (Ibid., 66), fazendo com que eles assimilar a ideia de que "a interface deveria facilitar as relações e as tarefas a serem realizadas" (Ibid., 66).

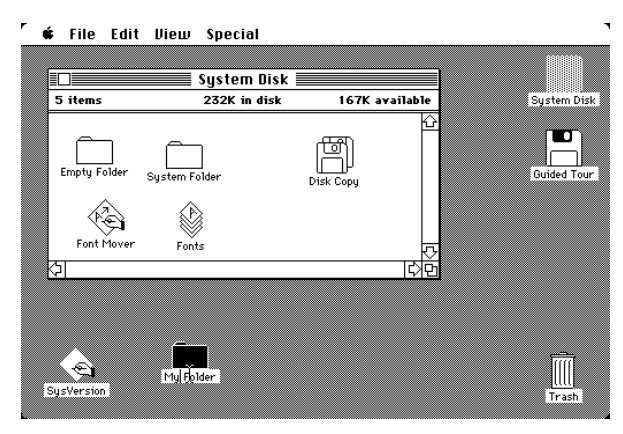

Figura 3 - Interface Apple Computer 1984.

Fonte: (http://zip.net/bsncB9)

O Windows 1.0, uma interface gráfica para o sistema operacional MS-DOS, foi lançado em 1985. No entanto, a interface adquiriu popularidade só em 1990 com o lançamento do Windows 3.0, identificado na figura 4. Entre as principais inovações da GUI, temos a possibilidade de maximizar a janela dos aplicativos e a utilização de atalhos para alternar entre os aplicativos maximizados. Quando nenhuma das aplicações executadas estava maximizada era possível alternar entre elas clicando em uma janela parcialmente visível, exatamente como em outras interfaces gráficas. 


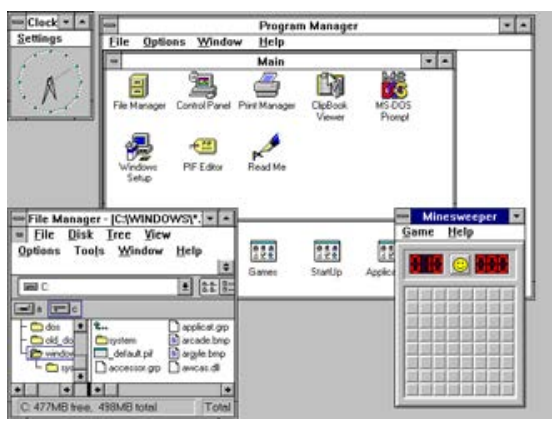

Figura 4 Interface do Windows 93.

Fonte: (http://zip.net/bkncp1)

A interface do Windows 10, lançada em julho de 2015, é um exemplo de evolução estética, como podemos observa na figura 5. A interface utiliza cores vibrantes e o conteúdo organizado em módulos, possuindo uma grande preocupação com o refinamento estético.

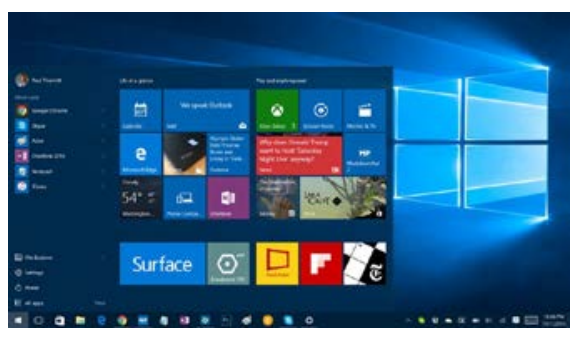

Figura 5 - Interface Windows 8.

Fonte: Captura de tela feito pela autora.

\subsection{Flat Design}

A evolução das interfaces gráficas acompanha o avanço da tecnologia, possibilitando que designers possam utilizar recursos para projetar uma melhor impressão estética ao usuário. Assim, surgem tendências estéticas que visam explorar os recursos tecnológicos em busca de maior interatividade.

A tendência estética utilizada nos últimos anos era o Skeuomorphism que ainda possuía relação com as primeiras GUls, utilizando um tratamento estético dos elementos que lembravam a forma e o visual do objeto no mundo real para que o usuário pudesse se familiarizar com a interface do computador. No entanto, de acordo com alguns designers ${ }^{3}$, o Skeuomorphism está ultrapassado e deve dar lugar a uma nova tendência que concilie forma, funcionalidade e tecnologia: o Flat Design.

O Flat Design é uma denominação para a recente tendência do design de interfaces, que tem sido discutida em blogs ${ }^{4}$ e fóruns ${ }^{5}$ da área. 0 flat, traduzido para

\footnotetext{
${ }^{3}$ Scott Thomas, The Noun Project; Allan Yu, SVPPLY; Robert Lenne, Artsy; Kyle Meyer, Facebook; Rus Yusupov, Big Human; Ian Storm Taylor, Segment.io; Pasquale D’Silva, Elepath; Stuart Regan, Finely; Jon Friis, Kera.io; Mig Reyes, 37Signals; Patrick Algrim.

Disponível em <http://layervault.tumblr.com/post/32267022219/flat-interface-design/>

${ }^{4}$ Chocola Design. Disponível em < http://chocoladesign.com/tendencias-de-ui-flat-design/>.

Gizmodo. Disponível em <http://gizmodo.uol.com.br/o-que-e-flat-design/>.
} 
português, significa plano. Atribui-se a sua popularização ao lançamento da interface da Metro UI, uma interface gráfica desenvolvida pela Microsoft baseada em princípios do design funcionalista, originalmente desenvolvimento para o uso no sistema operacional dos móvel da empresa (tablets e o smartphone da Microsoft, o Windows Phone) e posteriormente adotada para o sistema operacional para computadores (a partir do Windows 8). Esta interface surpreendeu os usuários por possuir uma aparência estética diferente do que vinha sendo utilizado em interfaces digitais. Acredita-se que simplificação das interfaces gráficas foi necessária, tendo em vista o tamanho e a resolução da tela dos dispositivos móveis, como smartphones e tablets.

A Metro UI é baseado nos princípios clássicos da escola suíça de design gráfico. De acordo com a Microsoft, o nome dado à linguagem de design representa toda a velocidade, autenticidade e modernidade utilizada, bem como a constante "movimentação" do seu código - o que nos leva a crer que ele esteja em constante desenvolvimento. Para o design visual, a Microsoft utilizou uma aparência estética mais limpa, excluindo elementos gráficos supérfluos ao sistema operacional. De acordo com a equipe de design da empresa, a tipografia utilizada nessa interface chamada Sergoe - foi inspirada nos símbolos existentes no sistema público de metrô de Londres. A Metro UI foi projetada para tornar a experiência dos usuários mais agradável e fluída; para isso, a interface utiliza botões e títulos grandes, ícones planos (figura e fundo), tipografia sem serifa e com variações de tamanho, além de transições mais suaves para a navegação entre as telas.

Na figura 6, é possível observar as mudanças do Windows 8 em comparação com o Windows 7: no Windows 7 os ícones são realistas, possuem sombra, gradientes, efeitos em 3D; já no Windows 8 os ícones são planos e com desenho simplificado, estão organizados dentro de blocos coloridos. Desta forma, pode-se dizer que o Windows 7 representa o Skeuomorphism e o Windows 8 o Flat Design.

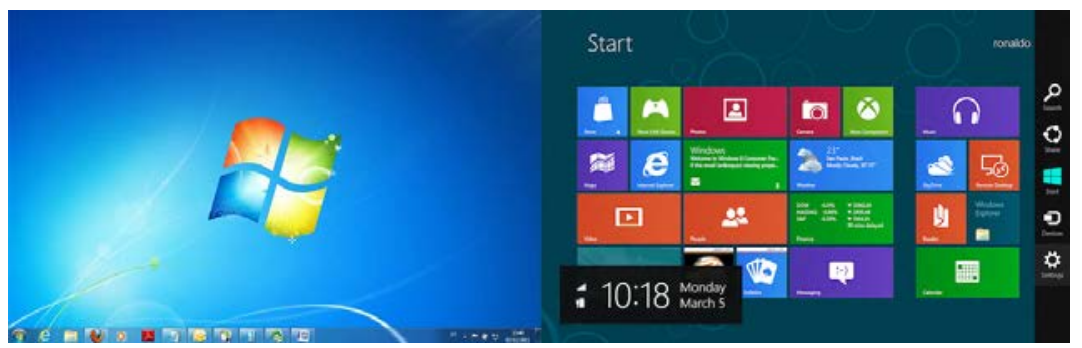

Figura 6 - Desktop Windows 7 e Windows 8, respectivamente.

Fonte: (http://zip.net/bvlBNT).

\subsection{Análise iOS6 e iOS7 no aplicativo "Notas"}

O iOS 6 foi o sistema operacional da Apple lançado em setembro de 2012 trazendo algumas mudanças para o usuário, tais como melhora da funcionalidade e aparência dos aplicativos. A cada atualização do sistema a empresa visa melhorar a

Dzestudio. Disponível em < http://www.dzestudio.com.br/blog/5-razoes-para-o-sucesso-do-flatdesign/>. UX.Blog. Disponível em: < http://www.uxdesign.blog.br/design-de-interfaces/flat-designrecultura-interface/>

${ }^{5}$ Branch. Disponível em: <http://branch.com/g/flat-design-convensions/> 
estética e a usabilidade da interface, possibilitando que o usuário possa usufruir de todos os recursos do seu dispositivo móvel. Diferentemente de atualizações anteriores, onde o usuário não percebia uma mudança drástica na interface, a atualização do iOS 6 para o iOS 7, lançado para os usuários em setembro de 2013, apresentou alterações significativas no design de interfaces, sendo alvo de críticas positivas e negativas no mercado. A versão mais recente do sistema é o iOS 9 que ainda mantém a mesma estética utilizada no iOS 7. Com a aparência mais limpa, o iOS 7 possui uma estética simplificada quando comparada a utilizada nas atualizações anteriores. Assim, a etapa de análise comparou a interface gráfica do iOS 6, que possui características do Skeuomorphism, com a interface gráfica do iOS 7, considerada como uma interface flat. Para a análise, foram utilizadas dois iPads: um com o sistema iOS6 e outro com o iOS7. A partir da observação entre os dois sistemas, analisou-se as mudanças relativas a estética, usabilidade e interação do aplicativo "Nota".

No iOS 6 o aplicativo de notas assemelhava-se a um caderno de couro com blocos de papel, como podemos visualizar na figura 7. A tipografia utilizada para o cadastro de novas notas era manuscrita, fazendo referência a caligrafia manual. Ao adicionar uma nova nota, a transição entre uma página e outra simulava o movimento de uma folha de papel. O menu do aplicativo era composto por uma barra superior marrom com textura de couro, que possuía ao centro o texto da nota adicionada, à esquerda o botão "Notes" que mostrava as notas cadastradas e à direita o botão "+" (adicionar) que adicionava uma nova nota. Os botões enviar, deletar e avançar eram localizados no rodapé do aplicativo e seu desenho possuía traços manuais.

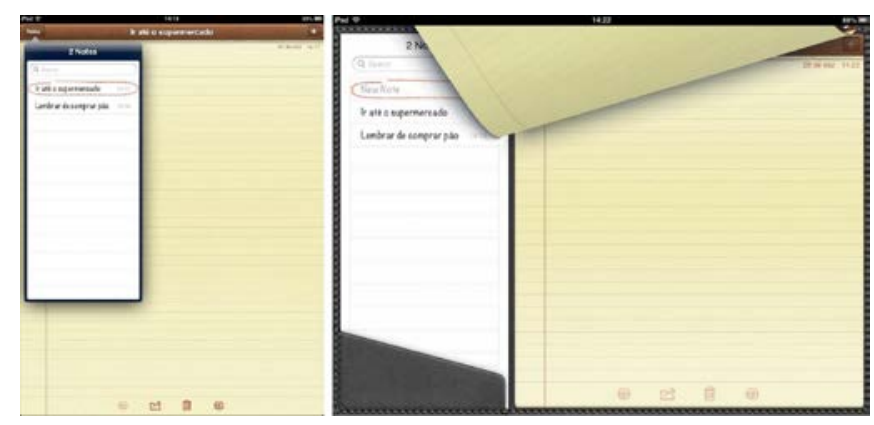

Figura 7 - Aplicativo Notas iOS6 versão horizontal.

Fonte: Captura de tela feita pela autora.

Na figura 7, podemos observar também o aplicativo na versão horizontal. Nesta orientação, o aplicativo mostrava dois blocos de papel: um em amarelo, para adicionar notas e outro em branco, contendo as notas já adicionadas. Uma aba de couro continha o bloco de papel branco com as notas já armazenadas e com o botão "search" (buscar). Para a seleção das notas armazenadas, utilizava-se um traço irregular e vermelho, assemelhando-se a um risco de caneta em um papel.

Todos os elementos utilizados na composição visual do aplicativo parecem ter o objetivo de torná-lo semelhante a um caderno de couro do mundo analógico, tornando-o extremamente realista: as sombras utilizadas davam ideia de profundidade, as texturas de couro e de papel e a borda costurada imitavam texturas físicas, os gradientes auxiliavam nos efeitos de claro e escuro conferindo maior realismo ao aplicativo. 
A figura 8 mostra a versão lançada no iOS 7, em que a interface é mudada drasticamente, deixando de fazer referência a um caderno de couro. O menu, localizado anteriormente em uma barra superior, foi simplificado: a barra e o botão "+" foram retirados. Agora, ao escrever a nota, ela é automaticamente armazenada no aplicativo, ou seja, não precisa mais ser adicionada. Os botões enviar, deletar e avançar foram colocados no topo do aplicativo, localizados à direita. O botão "Notes" continua localizado à esquerda e sua forma foi reduzida, ou seja, não existe mais uma caixa ou borda que indique que ele é um botão. Existe apenas a indicação pelo nome "Notes". Isso pode fazer com que o usuário não entenda que ele é um botão. A nova interface é composta por uma tela em branco e a tipografia utilizada é uma fonte bastão, a Helvetica. Observa-se uma sutil utilização de uma textura de papel no background do aplicativo. Para a seleção de notas armazenadas, utiliza-se um preenchimento amarelo, assemelhando-se a uma seleção de menus em um software.

Figura 8 - Aplicativo Notas iOS7 orientação vertical e horizontal.

Fonte: Captura de tela feita pela autora.

As sombras e gradientes foram retirados e deram lugar a uma interface mais simples, contendo as mesmas funcionalidades da interface do iOS 6 . No entanto, para um usuário que não estava familiarizado com a versão anterior, a nova interface pode parecer simples demais, fazendo com que haja dificuldade na interação.

\section{CONCLUSÃO}

O design de interfaces é uma atividade recente, portanto ainda não é possível definir a sua história de forma precisa, abrangendo todos os movimentos estéticos utilizados desde as primeiras interfaces gráficas do usuário. A análise de estéticas visuais utilizadas no passado auxilia no aperfeiçoamento das interfaces gráficas do futuro. A realização desta pesquisa foi um desafio: como buscar teorias para explicar uma terminologia de design de interfaces surgida na Internet? Para isso, foi preciso ir em busca de conhecimentos de outras áreas e realizar análises que pudessem qualificar teoricamente o trabalho.

O Skeuomorphism é uma estética visual que utiliza ornamentos ou detalhes para representar objetos da vida real. Esta tendência é caracterizada pelo uso de sombras, gradientes, texturas, efeitos 3D, chanfro, entre outros recursos visuais que tornem os elementos de interface mais semelhantes a sua forma do mundo real. 0 Skeuomorphism faz uso de metáfora e affordances, que referem-se à utilização das 
propriedades reais do objeto para mostrar indicações de como usá-lo. O Flat Design é uma tendência visual contemporânea caracterizada pela simplificação dos elementos de interface com foco na forma, funcionalidade e tecnologia. Para isso, esta tendência utiliza botões e títulos grandes, ícones planos, tipografias sem serifa com variações de tamanho e transições mais suaves para a navegação entre as telas.

A partir das bibliografias e as análises de estética visual de interfaces, foi possível identificar a influência do Flat Design no design de interfaces. Embora tenha sido tratada como uma tendência durante toda essa pesquisa, o flat parece estar atrelado a uma evolução no design de interfaces. Se no Skeuomorphism as interfaces se preocupavam em "parecer", utilizando uma quantidade de elementos visuais para fornecer metáforas e affordances, o Flat Design entende que grande parte do usuário já compreende os elementos de interface e preocupa-se em "informar", utilizando poucos elementos visuais com foco no considera importante: o conteúdo.

As análises evidenciam a influência da tendência do Flat Design no produto de duas grandes empresas, a Microsoft e a Apple. A transição para o Flat Design obteve popularidade com o anúncio da Metro UI da Microsoft, influenciando diversos designers a adotarem a tendência em seus projetos. A repercussão do Metro UI foi uma novidade no mercado, fazendo com que o Windows Phone se destacasse de seus principais concorrentes: o iOS e o Android. A Apple sentiu a necessidade de revitalizar seu sistema operacional, incorporando a tendência no iOS7. Desta forma, observa-se a influência de uma tendência no design de interfaces, fazendo com que duas grandes empresas mudem a aparência estética de seus consolidados sistemas operacionais.

Assim, conclui-se que a tendência difundiu-se de forma discursiva, a partir da utilização da estética flat pela Microsoft e, posteriormente, pela Apple. A partir da mudança drástica de design promovida pela Microsoft, observou-se um movimento contra o Skeuomorphism. Designers utilizaram blogs para propagar a ideia que de o Skeuomorphism está ultrapassado e o Flat Design é a nova tendência a ser seguida e utilizada no design de interfaces. Enquanto alguns estudiosos e profissionais acreditam que o flat é tendência datada, outros afirmam que é uma evolução do design de interfaces. Discussões a parte, é importante ressaltar que o Flat Design nem sempre pode ser a melhor alternativa para um projeto. Cada projeto possui as suas especificidades e características próprias. O flat é apenas uma possibilidade estética que pode ser explorada e não uma doutrina ou regra no design de interfaces da atualidade. Por fim, espera-se que os resultados deste trabalho possam contribuir para discussões acerca do aperfeiçoamento estético das interfaces gráficas, com o objetivo de descobrir melhores formas de transmitir informações, ideias e conceitos aos usuários. 


\section{REFERÊNCIAS}

CALDAS, Dario. Observatório de sinais: teoria e prática da pesquisa de tendências. Rio de Janeiro: Editora Senac, 2004.

LAKATOS, E. M.; MARCONI, M. Fundamentos de Metodologia Científica. São Paulo: Atlas, 2010.

NORMAN, Donald. O design do dia-a-dia. Rocco 2006.

PICOLI, Julia Isoppo. Coolhunting: pesquisador e suas metodologias. São Paulo:

EnModa, 2009. Disponível na Internet por http em:

<https://www.enmoda.com.br/site/pesquisas/lista.asp?menu_codigo=1>Acesso em:

10 ago 2016

RAYMOND, Martin. The Trend Forecaster's Handbook. Londres: Laurence King, 2010.

ROYO, J. P. Design Digital. São Paulo: Rosari, 2008.

VEJLGAARD, Henrik. Anatomy of a Trend (e-book). New York: Confetti Publishing at Smashwords, 2007 (e-book). 